ISSN 1561-2430 (Print)

ISSN 2524-2415 (Online)

УДК $517.44 ; 517.58 ; 51-72 ; 239.199$

https://doi.org/10.29235/1561-2430-2020-56-4-496-503

Поступила в редакцию 11.07.2019

Received 11.07.2019

\author{
А. В. Батяновский ${ }^{1}$, В. А. Намиот ${ }^{2}$, И. В. Филатов ${ }^{3}$, \\ В. Г. Туманян ${ }^{4}$, Н. Г. Есипова ${ }^{4}$, И. Д. Волотовский ${ }^{1}$ \\ ${ }^{1}$ Институт биофизики клетки и клеточной инженерии \\ Национальной академии наук Беларуси, Минск, Беларусь \\ ${ }^{2}$ Научно-исследовательский институт ядерной физики Московского государственного университета \\ им. М. В. Ломоносова, Москва, Россия \\ ${ }^{3}$ Московский физико-технический институт, Долгопрудный, Россия \\ ${ }^{4}$ Институт молекулярной биологии им. В. А. Энгельгардта \\ Российской академии наук, Москва, Россия

\section{ФУРЬЕ-ПРЕОБРАЗОВАНИЕ В СФЕРИЧЕСКИХ СИСТЕМАХ КООРДИНАТ КАК ИНСТРУМЕНТ РЕШЕНИЯ ЗАДАЧ СТРУКТУРНОЙ БИОЛОГИИ}

\begin{abstract}
Аннотация. Рассмотрено применение наиболее распространенной адаптации Фурье-анализа в сферических системах координат для решения ряда задач структурной биологии, а именно: разложения по плоским волнам (при этом плоские волны представляются в виде разложения по сферическим функциям). Приводятся аргументы в пользу этого разложения в сравнении с другими разложениями по суперпозициям специальных функций. Получено более общее обоснование корректности данного разложения, чем существующие в настоящее время. Предложен способ представления групп атомов в виде Фурье-объекта и рассмотрены его возможности. Обсуждаются перспективы применения Фурье-анализа в структурной биофизике.

Ключевые слова: Фурье-анализ, функции Бесселя, вычислительно обоснованный докинг и сравнение белковых глобул, разложение по специальным функциям математической физики

Для цитирования. Фурье-преобразование в сферических системах как инструмент решения физических задач структурной биологии / А. В. Батяновский [и др.] // Вес. Нац. акад. навук Беларусі. Сер. фіз.-мат. навук. - 2020. - Т. 56, № 4. - C. 496-503. https://doi.org/10.29235/1561-2430-2020-56-4-496-503
\end{abstract}

\author{
Alexander V. Batyanovskii ${ }^{1}$, Vladimir A. Namiot ${ }^{2}$, Ivan V. Filatov ${ }^{3}$, Vladimir G. Tumanyan ${ }^{4}$, \\ Natalia G. Esipova ${ }^{4}$, Igor D. Volotovsky ${ }^{1}$ \\ ${ }^{I}$ Institute of Cell Biophysics and Cell Technology of the National Academy of Sciences of Belarus, Minsk, Belarus; \\ ${ }^{2}$ Institute of Nuclear Physics of the Lomonosov Moscow State University, Moscow, Russia \\ ${ }^{3}$ Moscow Institute of Physics and Technology, Dolgoprudny, Russia \\ ${ }^{4}$ Engelhard Institute of Molecular Biology of the Russian Academy of Sciences, Moscow, Russia
}

\title{
FOURIER TRANSFORMATION IN SPHERICAL SYSTEMS AS A TOOL OF STRUCTURAL BIOLOGY
}

Abstract. Applications of the most common adaptation of Fourier analysis in spherical coordinate systems used to solve a number of problems in structural biology, namely, flat wave decomposition (flat waves are represented as spherical functions decomposition), are herein considered. Arguments in favor of this decomposition are compared with other decompositions in superposition of special functions. A more general justification for the correctness of this decomposition is obtained than that existing today. A method for representing groups of atoms in the form of a Fourier object is proposed. It is also considered what opportunities give such a representation. The prospects for the application of Fourier analysis in structural biophysics are discussed.

Kewwords: Fourier analysis, Bessel functions, computationally supporter docking and comparison of protein globules, decomposition in special functions of mathematical physics

For citation. Batyanovskii A. V., Namiot V. A., Filatov I. V., Tumanyan V. G., Esipova N. G., Volotovsky I. D. Fourier transformation in spherical systems as a tool of structural biology. Vestsi Natsyianal'nai akademii navuk Belarusi. Seryia fizika-matematychnykh navuk = Proceedings of the National Academy of Sciences of Belarus. Physics and Mathematics series, 2020, vol. 56, no. 4, pp. 496-503 (in Russian). https://doi.org/10.29235/1561-2430-2020-56-4-496-503

Фурье-анализ является одним из самых распространенных методов интегральных преобразований, который широко применяется при анализе сложных процессов и пространственных структур, в первую очередь при анализе сигналов и явлений волновой природы. Кроме того, он 
обладает рядом важных математических и вычислительных особенностей, позволяющих получать или упрощать решение математических задач, связанных с дифференцированием и интегрированием, в частности, реализовывать на его основе вычисления in silico. Однако подобное применение Фурье-анализа требует использования специальных приемов по адаптации его базисного аппарата при поиске решения конкретных задач.

В предлагаемой статье рассмотрены особенности адаптации Фурье-анализа к задачам современной структурной биологии, проведено подтверждение ее основного принципа и обсуждены несовершенства и пробелы, предложен вариант получения Фурье-образа многоатомного объекта, проанализированы некоторые приемы работы с такими объектами и изучена применимость и перспективы Фурье-анализа в молекулярной биологии.

В настоящее время опубликовано значительное количество работ по представлению макромолекулярных объектов в виде разложений по комбинациям различных специальных функций [1-7]. Обычно эти подходы преследуют цели, связанные с оценкой пространственной симметрии в белковых структурах [2], сравнением профилей поверхностей молекул $[3,4]$ или же решением задач поиска мест посадки / избирательного контакта [5-7]. В таких задачах для быстрых пересчетов возможных вариантов взаимной ориентации весьма привлекательными являются методы, основанные на использовании сферических систем координат и сферических гармоник как специальных функций разложения исходных функций.

Наиболее перспективным в этом направлении является Фурье-разложение в силу ряда математических свойств Фурье-объектов и возможностей Фурье-анализа. В этом качестве Фурьеразложения в сферических координатах предлагается разложение по плоским волнам (пространственным компонентам стоячих плоских волн), суть которого сводится к тому, что коль скоро в решение волнового уравнения входит пространственная функция

$$
e^{-2 \pi i \vec{r} r}
$$

то решение такого уравнения в сферических координатах является формой Фурье для него. Следует отметить ряд исследований, в которых в сферических координатах производятся разложения по комбинациям сферических гармоник, отвечающих за угловые координаты, с самыми разными функциями, отвечающими за радиальную компоненту. Следует упомянуть полиномы Лежандра, различные вариации функций Лагерра, полиномы Цернике и т. д. Более того, подобные разложения нередко продолжают именовать Фурье-преобразованием, хотя весьма сомнительно, что их можно каким-либо образом свести к Фурье-преобразованию в сферических системах координат.

Для последующего рассмотрения будем использовать соотношение [1]

$$
F\left(k_{r}, k_{\theta}, k_{\varphi}\right)=\int_{0}^{\infty} r^{2} d r \int_{0}^{\pi} \sin \theta d \theta \int_{0}^{2 \pi} e^{2 \pi i r k\left(\cos \theta \cos k_{\theta}+\sin \theta \sin k_{\theta} \cos \left(\varphi-k_{\varphi}\right)\right)} f(r, \theta, \varphi) d \varphi,
$$

связывающее исходную функцию в сферических координатах $f(r, \theta, \varphi)$ и ее Фурье-образ $F\left(k_{r}, k_{\theta}, k_{\varphi}\right)$, зависящий от волнового вектора, который в свою очередь выражен опять же в сферических координатах.

Формула (1) пригодна для аналитического и прикладного использования лишь для узкого круга задач. Если при применении прямоугольных систем координат трехкратный интеграл 3D Фурье-преобразования существенно упрощен тем, что ядро преобразования позволяет разделять переменные, то в случае криволинейных координат использование таких интегралов, как правило, существенно осложнено, а применение ограничено. Для упрощения в случае использования сферических координат нами рассматривается вариант, когда исходная функция представима в виде произведения радиальной и угловой составляющих. Как показано в работе [8] (см. также [1] и Приложение к настоящей статье), важным для рассматриваемой задачи является соотношение 


$$
\begin{gathered}
J_{n+\frac{1}{2}}(z)= \\
=(-i)^{n} \sqrt{\frac{z}{2 \pi^{2}}} \sum_{m=-n}^{n}(-1)^{m} P_{n}^{m}\left(\cos k_{\theta}\right) e^{-i m k_{\varphi}} \int_{0}^{\pi} \int_{0}^{2 \pi} \sin \theta e^{i z\left(\cos \theta \cos k_{\theta}+\sin \theta \sin k_{\theta} \cos \left(\varphi-k_{\varphi}\right)\right)} P_{n}^{-m}(\cos \theta) e^{i m \varphi} d \varphi d \theta,
\end{gathered}
$$

где $P_{n}^{m}\left(\cos k_{\theta}\right) e^{-i m k_{\varphi}}$ и $P_{n}^{-m}\left(\cos k_{\theta}\right) e^{i m k_{\varphi}}-$ ненормированные сферические и комплексно-сопряженные им функции соответственно.

Полагая возникшие при выводе уравнения (2) переменные $k_{\varphi}$ и $k_{\theta}$ азимутальной и полярной компонентами волнового вектора, а также в результате сравнения (2) с (1), выявляется способ выражения Фурье-объектов в сферической системе координат в виде разложения по комбинациям сферических функций и функций Бесселя полуцелого порядка. Согласно (1) и (2), если исходная функция представима в виде разложения по сферическим функциям с коэффициентами $C_{m, n}(r)$, то Фурье-образ также представим в таком виде, но с коэффициентами

$$
4 \pi(-i)^{n} \int_{0}^{\infty} C_{n,-m}(r) J_{n+1 / 2}(k r) r^{2} d r
$$

что в случае сферической симметрии исходной функции приводит к известной формуле Фурьепреобразования сферически-симметричной функции. Сравнивая представленный вывод с обычными подходами, исходящими из возможности разложения $e^{-2 \pi i \vec{k} r}$ по комбинациям соответствующих функций [8], отметим идентичность результатов, хотя случай разложения по плоским волнам требует для приведения к форме Фурье-преобразования еще и использования свойств ортогональности сферических гармоник и функций Бесселя.

Из подобных представленному выводу можно упомянуть также варианты, изложенные в образовательных курсах $[9,10]$, однако они не учитывают угловые координаты, и поэтому их результаты ограничиваются лишь случаями, где исходные функции зависят только от радиуса.

С учетом обобщения известного выражения для энергии электростатического взаимодействия открываются возможности решения физических задач в Фурье-представлении с отличными от координатного представления вычислительной сложностью и идеологией:

$$
U=\int \frac{F_{1}(q) F_{2}(q)}{q^{2}} d^{3} q
$$

где $F_{1}(q)$ и $F_{2}(q)$ - Фурье-образы, $q$ - волновой вектор, $U$ - энергия взаимодействия.

В случае со структурной биологией все это может оказаться необходимым, поскольку вычисления в координатном представлении по огромному числу частиц и вариантов взаимного расположения требуют больших вычислительных мощностей даже при низкой точности, что чаще всего приводит к неустойчивым решениям.

Отметим, что Фурье-анализ в структурной биологии применяется либо исключительно для ускорения расчетов, либо для получения формальных пространственных характеристик в рамках задач сравнительного анализа. Данное разложение по таким специальным функциям позволяет перейти к некоторому аналогу дискретного Фурье-преобразования, который ограничен в силу отсутствия представления об атомарном устройстве макромолекул, для которого можно применять некоторые обобщения быстрых Фурье-преобразований с целью быстрого пересмотра различных взаимных расположений. Использование коэффициентов разложения для примерной оценки корреляции дает возможность получить альтернативный инструмент, к примеру, для избирательной молекулярной стыковки, сравнимый по времени и качеству с подходами, основанными на координатном представлении, но при неизбежной потере наглядности. Просматриваются перспективы конструирования из этих коэффициентов поворотных инвариантов с целью «картографирования» молекулярных поверхностей опять же с потерей наглядности. 
В связи с этим речь больше идет о задаче схожести и определении возможного взаимодействия по подобию.

Формулирование задач о трехмерном меж- и внутримолекулярном взаимодействиях в случае биомакромолекул со степенями и иерархиями регулярностей, а также о соответствии структурированных частей и целого может быть реальным в более общем Фурье-анализе с учетом атомарного устройства, локальных конформаций и укладки цепей.

Для демонстрации математических приемов, возникающих в таких задачах, рассмотрим пример поиска оптимального наложения атомарных структур. Самый простой вариант решения задачи - использование представления атомов как сфер различных радиусов. Фурье-образ от сферы, согласно соотношениям (1) и (2), имеет вид:

$$
\begin{gathered}
F\left(k_{r}, k_{\theta}, k_{f}\right)= \\
=\int_{0}^{R} r^{2} d r \int_{0}^{\pi} \sin \theta d \theta \int_{0}^{2 \pi} e^{2 \pi i r k\left(\cos \theta \cos k_{\theta}+\sin \theta \sin k_{\theta} \cos \left(\varphi-k_{\varphi}\right)\right)} d \varphi=2 \pi \int_{0}^{R} \frac{J_{1 / 2}(2 \pi r k)}{\sqrt{k r}} r^{2} d r= \\
=J_{3 / 2}(2 \pi R k)\left(\frac{R}{k}\right)^{3 / 2},
\end{gathered}
$$

где $R$ - радиус сферы, $k$ - длина волнового вектора. Фурье-образ ансамбля сфер с использованием суммирования векторов можно представить как

$$
F(\vec{k})=\sum_{n} e^{-2 \pi \vec{k} \vec{r}_{n}} J_{3 / 2}\left(2 \pi k R_{n}\right)\left(\frac{R_{n}}{k}\right)^{3 / 2},
$$

где $R_{n}$ - радиус $n$-го атома, $\overline{r_{n}}$ - вектор к центру $n$-го атома, $\bar{k}$ - волновой вектор. При этом выполнять преобразование, соответствующее параллельному переносу в сферических системах координат (ССК), можно по следующим выражениям:

$$
\begin{gathered}
R^{\prime}=\sqrt{R^{2}+R_{t}^{2}-2 R R_{t}\left(\sin \theta \sin \theta_{t} \cos \left(\varphi-\varphi_{t}\right)+\cos \theta \cos \theta_{t}\right)}, \\
\tan \varphi^{\prime}=\frac{R_{t} \sin \varphi_{t}-R \sin \varphi}{R_{t} \cos \varphi_{t}-R \cos \varphi}, \\
\left(R^{\prime} \cos \theta^{\prime}\right)^{2}=\left(R \cos \theta-R_{t} \cos \theta_{t}\right)^{2},
\end{gathered}
$$

где $(R, \theta, \varphi)$ - исходные координаты в ССК, $\left(R_{t}, \theta_{t}, \varphi_{t}\right)$ - координаты вектора переноса, $\left(R^{\prime}, \theta^{\prime}, \varphi^{\prime}\right)$ новые координаты.

Согласно аналогу теоремы о свертке в Фурье-анализе [11, гл. 6], пространственная корреляция двух атомарных структур может быть представлена через их Фурье-образы:

$$
K\left(\vec{r}^{\prime}\right)=\int f_{1}^{*}(\vec{r}) f_{2}\left(\vec{r}+\vec{r}^{\prime}\right) \overrightarrow{d r}=\int F_{1}^{*}(\vec{k}) F_{2}(\vec{k}) e^{2 \pi \vec{k} \vec{r}^{\prime}} \overrightarrow{d k}
$$

С учетом (4) при использовании двойного интеграла Гегенбауэра типа Пуассона [8, гл. 3.33], получим

$$
\begin{aligned}
K\left(r^{\prime}\right) & =\sum_{n} \sum_{m} \int_{0}^{\infty} d k k^{2} J_{3 / 2}\left(2 \pi k R_{n}\right)\left(\frac{R_{n}}{k}\right)^{3 / 2} J_{3 / 2}\left(2 \pi k R_{n}\right)\left(\frac{R_{m}}{k}\right)^{3 / 2} \int_{0}^{\pi} \int_{0}^{2 \pi} \sin k_{\theta} e^{-2^{*} \pi \vec{k}\left(\vec{r}_{m}-\vec{r}_{n}-\vec{r}^{\prime}\right)} d k_{\varphi} d k_{\theta}= \\
& =2 \pi \sum_{n} \sum_{m} \int_{0}^{\infty} d k k^{2} J_{3 / 2}\left(2 \pi k R_{n}\right)\left(\frac{R_{n}}{k}\right)^{\frac{3}{2}} J_{3 / 2}\left(2 \pi k R_{n}\right)\left(\frac{R_{m}}{k}\right)^{\frac{3}{2}} \frac{J_{\frac{1}{2}}\left(2 \pi k\left|\vec{r}_{m}-\vec{r}_{n}-\vec{r}^{\prime}\right|\right)}{\left(k\left|\vec{r}_{m}-\vec{r}_{n}-\vec{r}^{\prime}\right|\right)^{1 / 2}},
\end{aligned}
$$


где $\vec{r}_{n}$ - радиус-вектор к $n$-му атому первой структуры, $\vec{r}_{m}$ - радиус-вектор к $m$-му атому второй структуры, $R_{n}, R_{m}$ - радиусы сфер, представляющих эти атомы, $k, k_{\theta}, k_{\varphi}$ - координаты волнового вектора в ССК. Подынтегральное выражение в (5) содержит три функции Бесселя. Произведение двух функций Бесселя одного порядка можно преобразовать, согласно интегралу, полученному в [8, гл. 11.41]:

$$
\int_{0}^{\pi} \frac{\mathcal{C}_{\mathrm{v}}\left\{\sqrt{Z^{2}+z^{2}-2 Z z \cos \varphi}\right\}}{\left(Z^{2}+z^{2}-2 Z z \cos \varphi\right)^{1 / 2 v}} \sin ^{2 v} \varphi d \varphi=2^{v} \Gamma\left(v+\frac{1}{2}\right) \Gamma\left(\frac{1}{2}\right) \frac{\mathcal{C}_{\mathrm{v}}(Z)}{Z^{\mathrm{v}}} \frac{J_{\mathrm{v}}(z)}{z^{\mathrm{v}}},
$$

где $\mathcal{C}_{v}$ - произвольная цилиндрическая функция, Г - гамма-функция. Подставляя (6) в (5) и меняя порядок интегрирования, получим

$$
K\left(r^{\prime}\right)=\sum_{n} \sum_{m} 2 \pi^{2} R_{n}^{3} R_{m} \int_{0}^{\pi} \sin ^{3} \varphi d \varphi \int_{0}^{\infty} d k \cdot k^{2} \frac{J_{\frac{3}{2}}\left(2 \pi k \sqrt{R_{n}^{2}+R_{m}{ }^{2}-2 R_{n} R_{m} \cos \varphi}\right)}{\left(k \sqrt{R_{n}^{2}+R_{m}{ }^{2}-2 R_{n} R_{m} \cos \varphi}\right)^{3 / 2}} \frac{J_{\frac{1}{2}}\left(2 \pi k R_{n m}\right)}{\left(k R_{n m}\right)^{1 / 2}},
$$

где $R_{n m}=\left|\vec{r}_{m}-\vec{r}_{n}-\vec{r}^{\prime}\right|$. Несобственный интеграл по $k$ в (7) является вариантом частного случая разрывного интеграла [8, гл. 13.42]:

$$
\int_{0}^{\infty} J_{v}(a t) J_{v-1}(b t) d t=\left\{\begin{array}{c}
\frac{b^{v-1}}{a^{v}}, b<a, \\
\frac{1}{2 b}, b=a \\
0, b>a .
\end{array}\right.
$$

В результате искомая пространственная корреляция приводится к следующему виду:

$$
K\left(\vec{r}^{\prime}\right)=\sum_{n} \sum_{m} \pi R_{n}^{3} R_{m}^{3} \int_{\Theta} \frac{\sin ^{3} \varphi}{\left(\sqrt{R_{n}^{2}+R_{m}^{2}-2 R_{n} R_{m} \cos \varphi}\right)^{3}} d \varphi
$$

где $\Theta$ - пересечение отрезка $[0, \pi]$ и области, в которой выполняется условие

$$
\sqrt{R_{n}^{2}+R_{m}^{2}-2 R_{n} R_{m} \cos \varphi}<R_{n m}
$$

если таковая есть.

Последний интеграл в (8) берется при замене переменной интегрирования $\varphi$ на $\cos \varphi$. Подобное выражение, вероятно, возможно получить и без применения Фурье-анализа - через перекрывающиеся объемы сферических сегментов при наложении структур. С другой стороны, для более сложных задач (к примеру, поиска экстремумов корреляции или в случае представления атомарных плотностей более сложными комбинациями сферических функций) продемонстрированный подход явно предпочтительнее.

В заключение отметим, что простота преобразований Фурье-образов, соответствующих трансляционным смещениям в пространстве координат, наличие ротационных инвариантов, таких как характеры неприводимых представлений групп вращений и функций, зависящих от угла между векторами и разлагаемых по биполярным гармоникам, обеспечивает высокие вычислительные преимущества использования сферических функций и Фурье-объектов в задачах, для которых «узким местом» является взаимное расположение взаимодействующих объектов. 


\section{Список использованных источников}

1. Математический аппарат, включающий Фурье преобразование (разложение по плоским волнам с использованием сферических координат), позволяющий одновременно исследовать сложные перемещения, в том числе повороты и смещения, в сложных молекулярных конструкциях / А. В. Батяновский [и др.] // Биофизика. - 2019. - Т. 64, № 2.- C. 239-242. https://doi.org/10.1134/s0006302919020030

2. A Fourier analysis of symmetry in protein structure / W. R. Taylor [et al.] // Protein Engineering, Design and Selection. - 2002. - Vol. 15, № 2. - P. 79-89. https://doi.org/10.1093/protein/15.2.79

3. SaberiFathi, S. M. Geometrical comparison of two protein structures using Wigner-D functions / S. M. SaberiFathi, D. T. White, J. A. Tuszynski // Proteins. - 2014. - Vol. 82, № 10. - P. 2756-2769. https://doi.org/10.1002/prot.24640

4. Mavridis, L. 3D-blast: 3D protein structure alignment, comparison, and classification using spherical polar Fourier correlations / L. Mavridis, D. W. Ritchie // Pacific Symposium on Biocomputing 2010: proc. Int. conf. - Hawaii, 2010. P. 281-292. https://doi.org/10.1142/9789814295291_0030

5. PIPER: an FFT-based protein docking program with pairwise potentials / D. Kozakov [et al.] // Proteins: Structure, Function, and Bioinformatics. - 2006. - Vol. 65, № 2. - P. 392-406. https://doi.org/10.1002/prot.21117

6. The ClusPro web server for protein-protein docking / D. Kozakov [et al.] // Nature Protocols. - 2017. - Vol. 12, № 2. P. 255-278. https://doi.org/10.1038/nprot.2016.169

7. Bajaj, C. F2Dock: fast Fourier protein-protein docking / C. Bajaj, R. Chowdhury, V. Siddavanahalli // IEEE/ACM Trans. Comput. Biol. Bioinformatics. - 2011. - Vol. 8, № 1. - P. 45-58. https://doi.org/10.1109/tcbb.2009.57

8. Watson, G. N. A Treatise on the Theory of Bessel Functions / G. N. Watson. - Cambridge: University press, 1922. $804 \mathrm{p}$.

9. Qing Wang. Fourier Analysis in Polar and Spherical Coordinates [Electronic resource] / Qing Wang, O. Ronneberger, H. Burkhardt. - 2008. - Mode of access: https://lmb.informatik.uni-freiburg.de/Publications/2008/WRB08/wa_report01_08. pdf. - Date of access: 23.07.2019.

10. Radial functions and the Fourier transform [Electronic resource] // Notes for Math 583A (2008). - Mode of access: https://www.math.arizona.edu/ faris/methodsweb/hankel.pdf. - Date of access: 23.07.2019.

11. Bracewell, R. N. The Fourier Transform and Its Applications / R. N. Bracewell. - McGraw-Hill, 2000. - 640 p.

\section{Referencies}

1. Batyanovskii A. V., Namiot V. A., Filatov I. V., Esipova N. G., Volotovskii I. D. A Mathematical Approachtha tIncludes the Fourier Transform (Plane Wave sDecomposition Using Spherical Coordinates) for the Study of Simultaneously Complex Movementsin Particular Rotationsand Displacementsin Complicated Molecular Constructions. Biofizika = Biophysics, 2019, vol. 64, no. 2, pp. 239-242 (in Russian). https://doi.org/10.1134/s0006302919020030

2. Taylor W. R., Heringa J., Baud F., Flores T. P. A Fourier analysis of symmetry in protein structure. Protein Engineering, Design and Selection, 2002, vol. 15, no. 2, pp.79-89. https://doi.org/10.1093/protein/15.2.79

3. SaberiFathi S. M., White D. T., Tuszynski J. A. Geometrical comparison of two protein structures using Wigner-D functions. Proteins: Structure, Function, and Bioinformatics, 2014, vol. 82, no. 10, pp. 2756-2769. https://doi.org/10.1002/ prot. 24640

4. Mavridis L., Ritchie D. W. 3D-blast: 3D protein structure alignment, comparison, and classification using spherical polar Fourier correlations. Proceedings international conference "Pacific Symposium on Biocomputing 2010”. Hawaii, 2010, pp. 281-292. https://doi.org/10.1142/9789814295291_0030

5. Kozakov D., Brenke R., Comeau S. R., Vajda S. PIPER: an FFT-based protein docking program with pairwise potentials. Proteins: Structure, Function, and Bioinformatics, 2006, vol. 65, no. 2, pp. 392-406. https://doi.org/10.1002/prot.21117

6. Kozakov D., Hall D. R., Bing Xia, Porter K. A., Padhorny D., Yueh C., Beglov D., Vajda S. The ClusPro web server for protein-protein docking. Nature Protocols, 2017, vol. 12, no. 2, pp. 255-278. https://doi.org/10.1038/nprot.2016.169

7. Bajaj C., Chowdhury R., Siddavanahalli V. F2Dock: fast Fourier protein-protein docking. IEEE/ACM Transactions on Computational Biology and Bioinformatics, 2011, vol. 8, no. 1, pp. 45-58. https://doi.org/10.1109/tcbb.2009.57

8. Watson G. N. A Treatise on the Theory of Bessel Functions. Cambridge, The University Press, 1922.804 p.

9. Qing Wang, Ronneberger O., Burkhardt H. Fourier Analysis in Polar and Spherical Coordinates. Technical Report 1, IIF-LMB, Computer Science Department, University of Freiburg (2008). Available at: https://lmb.informatik.uni-freiburg.de/ Publications/2008/WRB08/wa_report01_08.pdf (accessed 23.07.2019).

10. Radial functions and the Fourier transform. Notes for Math $583 \mathrm{~A}$ (2008) Available at: https://www.math.arizona. edu/ faris/methodsweb/hankel.pdf (accessed 23.07.2019).

11. Bracewell R. N. The Fourier Transform and Its Applications. McGraw-Hill, 2000. 640 p.

Приложение

Исходим из частного случая $v=1 / 2$ гегенбауэровского обобщения интеграла Пуассона $[8$, гл. 3.32]:

$$
J_{n+1 / 2}(z)=(-i)^{n} \sqrt{\frac{z}{2 \pi}} \int_{0}^{\pi} e^{i z \cos (\varphi)} P_{n}(\cos (\varphi)) \sin (\varphi) d \varphi
$$


где $J_{n+1 / 2}(z)$ - функция Бесселя полуцелого порядка, $P_{n}(x)$ - полином Лежандра.

Первая часть доказательства проведена подобно тому, как в [8, гл. 3.33] проводится доказательство двойного интеграла Гегенбауэра типа Пуассона. Рассмотрим очевидное следствие интеграла (П.1):

$$
J_{n+1 / 2}(z)=(-i)^{n} \sqrt{\frac{z}{2 \pi^{3}}} \int_{0}^{\pi} e^{\mathrm{iz} \cos (\theta)} P_{n}(\cos (\theta)) \sin (\theta) d \theta \int_{0}^{\pi} \sin ^{0}(\varphi) d \varphi,
$$

где $\sin$ в нулевой степени записан для согласования с доказательством в указанном источнике.

На следующем шаге, рассматривая угловые координаты как широту и долготу, с переходом в декартову систему координат, обозначив направляющие косинусы вектора интегрирования как

$$
[L=\cos (\varphi) \sin (\theta), M=\sin (\varphi) \sin (\theta), N=\cos (\theta)]
$$

и учитывая, что элемент поверхности

$$
d \Omega=\sin (\theta) d \theta d \varphi
$$

приводим (П.2) к следующему виду:

$$
J_{n+1 / 2}(z)=(-i)^{n} \sqrt{\frac{z}{2 \pi}} \frac{1}{\pi} \iint_{M \geq 0} e^{i z N} P_{n}(N) M^{0} d \Omega .
$$

Проведя циклическую перестановку орт, получим

$$
J_{n+1 / 2}(z)=(-i)^{n} \sqrt{\frac{z}{2 \pi}} \frac{1}{\pi} \iint_{N \geq 0} e^{i z L} P_{n}(L) N^{0} d \Omega
$$

или

$$
J_{n+1 / 2}(z)=(-i)^{n} \sqrt{\frac{z}{2 \pi}} \frac{1}{\pi} \int_{0}^{\pi / 2} \int_{0}^{2 \pi} e^{i z \cos \varphi \sin \theta} P_{n}(\cos \varphi \sin \theta) \sin \theta d \varphi d \theta .
$$

С учетом того, что в (П.3) один из интегралов охватывает полный период подынтегральной функции по координате $\varphi$, можем поступить так:

$$
\begin{gathered}
J_{n+1 / 2}(z)=(-i)^{n} \sqrt{\frac{z}{2 \pi}} \frac{1}{\pi} \int_{0}^{\pi / 2} \int_{0}^{2 \pi} e^{i z \cos \left(\varphi-k_{\theta}\right) \sin (\theta)} P_{n}\left(\cos \left(\varphi-k_{\theta}\right) \sin (\theta)\right) \sin (\theta) d \varphi d \theta \\
J_{n+\frac{1}{2}}(z)=(-i)^{n} \sqrt{\frac{z}{2 \pi^{2}}} \int_{0}^{\pi / 2 \pi} \int_{0}^{2 \pi} e^{i z\left(\cos \varphi \cos k_{\theta}-\sin \varphi \sin k_{\theta}\right) \sin \theta} P_{n}\left(\left(\cos \varphi \cos k_{\theta}-\sin \varphi \sin k_{\theta}\right) \sin \theta\right) \sin \theta d \varphi d \theta .
\end{gathered}
$$

Снова повторяя предыдущие операции по циклической перестановке орт, получим

$$
\begin{gathered}
J_{n+\frac{1}{2}}(z)=(-i)^{n} \sqrt{\frac{z}{2 \pi^{2}}} \iint_{N \geq 0} e^{i z\left(L \cos k_{\theta}-M \sin k_{\theta}\right)} P_{n}\left(L \cos k_{\theta}+M \sin k_{\theta}\right) d \Omega, \\
J_{n+\frac{1}{2}}(z)=(-i)^{n} \sqrt{\frac{z}{2 \pi^{2}}} \iint_{M \geq 0} e^{i z\left(N \cos k_{\theta}-L \sin k_{\theta}\right)} P_{n}\left(N \cos k_{\theta}+L \sin k_{\theta}\right) d \Omega, \\
J_{n+\frac{1}{2}}(z)=(-i)^{n} \sqrt{\frac{z}{2 \pi^{2}}} \int_{0}^{\pi} \int_{0}^{\pi} e^{i z\left(\cos \theta \cos k_{\theta}+\sin \theta \sin k_{\theta} \cos \varphi\right)} P_{n}\left(\cos \theta \cos k_{\theta}+\sin \theta \sin k_{\theta} \cos \varphi\right) \sin (\theta) d \varphi d \theta .
\end{gathered}
$$


Воспользуемся на данном этапе теоремой суммирования сферических гармоник, точнее, следующим ее видом:

$$
P_{n}\left(\cos \theta_{1} \cos \theta_{2}+\sin \theta_{1} \sin \theta_{2} \cos \varphi\right)=\sum_{m=-n}^{n}(-1)^{m} P_{n}^{-m}\left(\cos \theta_{1}\right) P_{n}^{m}\left(\cos \theta_{2}\right) \cos (m \varphi),
$$

где $P_{n}^{m}$ - обобщенный полином Лежандра

В итоге можно записать выражение (П.6) как

$$
\begin{gathered}
J_{n+\frac{1}{2}}(z)= \\
=(-i)^{n} \sqrt{\frac{z}{2 \pi^{2}}} \int_{0}^{\pi} \int_{0}^{\pi} e^{i z\left(\cos \theta \cos k_{\theta}+\sin \theta \sin k_{\theta} \cos \varphi\right)} \sum_{m=-n}^{n}(-1)^{m} P_{n}^{-m}(\cos \theta) P_{n}^{m}\left(\cos k_{\theta}\right) \cos (m \varphi) \sin (\theta) d \varphi d \theta .
\end{gathered}
$$

Вынося суммирование и множители, содержащие независимые переменные, за знак интеграла, и используя формулу Эйлера, получаем соотношение (2) основного текста.

\section{Информация об авторах}

Батяновский Александр Валерьевич - кандидат физико-математических наук, научный сотрудник, Институт биофизики и клеточной инженерии Национальной академии наук Беларуси (ул. Академическая, 27, 220072, г. Минск, Республика Беларусь). E-mail: alexandersn@ibp.org.by

Намиот Владимир Абрамович - доктор физико-математических наук, старший научный сотрудник, Научно-исследовательский институт ядерной физики им. Д. В. Скобельцына Московского государственного университета им. М. В. Ломоносова, (ул. Ленинские Горы, 1с2, 119991, г. Москва, Российская Федерация). E-mail: vnamiot@gmail.ru

Филатов Иван Васильевич - кандидат физико-математических наук, преподаватель, Московский физико-технический институт (Национальный исследовательский университет) (Институтский пер., 9, 141701, г. Долгопрудный, Российская Федерация).

E-mail: ivfilatov@mail.ru

Туманян Владимир Гайевич - доктор физико-математических наук, профессор, заведующий лабораторией вычислительных методов системной биологии, Институт молекулярной биологии им. В. А. Энгельгардта Российской академии наук (ул. Вавилова, 32, 117984, г. Москва, Российская Федерация). E-mail: tuman@eimb.ru

Есипова Наталья Георгиевна - кандидат физико-математических наук, старший научный сотрудник, Институт молекулярной биологии им. В. А. Энгельгардта Российской академии наук (ул. Вавилова, 32, 117984 , г. Москва, Российская Федерация). E-mail: nge.@eimb.ru

Волотовский Игорь Дмитриевич - академик Национальной академии наук Беларуси, доктор биологических наук, профессор, заведующий лабораторией молекулярной биологии клетки, Институт биофизики и клеточной инженерии Национальной академии наук Беларуси (ул. Академическая, 27, 220072, г. Минск, Республика Беларусь). E-mail: volot@biobel.bas-net.by

\section{Information about the authors}

Alexander V. Batyanovskii - Ph. D. (Physics and Mathematics), Researcher, Institute of Cell Biophysics and Cell Technology of the National Academy of Sciences of Belarus (27, Akademicheskaya Str., 220072, Minsk, Republic of Belarus).E-mail: alexandersn@ibp.org.by

Vladimir A. Namiot - Dr. Sc. (Physics and Mathematics), Senior Researcher, Lomonosov Moscow State University (1/2, Leninskie Gory, 119991, Moscow, Russian Federation).E-mail: vnamiot@gmail.ru

Ivan V. Filatov - Ph. D. (Physics and Mathematics), Teacher, Moscow Institute of Physics and Technology (9, Institutskii Lane, Dolgoprudny, 141701, Moscow Region, Russian Federation). E-mail: ivfilatov@mail.ru

Vladimir G. Tumanyan - Dr. Sc. (Physics and Mathematics), Professor, Head of the Laboratory of Computational Methods for System Biology, Engelhard Institute of Molecular Biology of the Russian Academy of Sciences (32, Vavilov Str., 119991, Moscow, Russian Federation). E-mail: tuman@eimb.ru

Natalia G. Esipova - Ph. D. (Physics and Mathematics), Senior Researcher, Engelhard Institute of Molecular Biology of the Russian Academy of Sciences (32, Vavilov Str., 119991, Moscow, Russian Federation). E-mail: nge.@eimb.ru

Igor D. Volotovsky - Academician of the National Academy of Sciences of Belarus, Dr. Sc. (Biology), Professor, Head of the Laboratory of Molecular Cell Biology, Institute of Cell Biophysics and Cell Technology of the National Academy of Sciences of Belarus (27, Akademicheskaya Str., 220072, Minsk, Republic of Belarus). E-mail: volot@biobel. bas-net.by 